\title{
Field Strength Estimation for Wireless Mobile Communication Based on Ray Tracing Technique \\ SEGGANI HIZIA ${ }^{1, a}$, Prof. GAO QIANG ${ }^{2, b}$
}

${ }^{1}$ Department of Electronic and Information Engineering, Beijing University of Aeronautics and Astronautics (BUAA). Beijing, China

${ }^{2}$ Department of Electronic and Information Engineering, Beijing University of Aeronautics and Astronautics (BUAA). Beijing, China

ahizia.seggani@gmail.com, b gaoqiang@buaa.edu.cn

Keywords: Mobile Communication, Ray Tracing, Grid Partitioning, Field Strength Estimation, Smoothing, Centered Moving Average.

\begin{abstract}
In the network planning and optimization of mobile communication, cellular signal strength estimate results not only determine the rationality of the planning results, but also affect the quality of the network optimization, so designing accurate signal strength estimation methods is the key problem of mobile communication network research .This paper presents a model to estimate field strength inside a campus using a ray tracing technique based on three-dimensional geometric theory, geometrical optics theory and the uniform theory of diffraction (UTD).Then a smoothing process which is centered moving average is further used to smooth the field strength distribution, and the simulation results are compared with measurement.
\end{abstract}

\section{Introduction}

The rapid growth of wireless communications involves efficient network planning of cellular mobile communication. The primary processes in the telecommunication network industry consist of network site identification, planning and signal strength measurements with coverage estimation for the extension of the system. Estimation methods based on empirical models [1] which can't be used to estimate in specific scenarios are not exact. Although ray tracing technique can track electromagnetic wave according to the scenario information; a common inverse tracing technique with high complexity is not suitable for the estimation of outdoor field strength. So an outdoor cellular field strength estimation method with high accuracy and low complexity needs to be designed.

In this paper, we describe a realization of the cell field strength estimation system software designed using Visual Studio as a debugging environment, $\mathrm{C}++$ as the development language, MapInfo as secondary development tools on MFC interface (Microsoft Foundation Classes), and SQL server to access the database. This system software is based on ray tracing which is suitable for outdoor scenes, and it is based on a method that divides the space into grids, records the grid number each ray passes, finds the intersection with buildings, and determines all the routes of ray tracing combined with iterative process, finally the field strength of each grid will be calculated. The ray tracing method is based on three-dimensional geometric theory, geometrical optics theory and the uniform theory of diffraction (UTD) [2].

\section{Simulation setup}

The first task of this paper is the implementation of cellular signal strength estimation system interface [3] in English version figure1. The system consists of three parts: data storage and the processing subsystem, the signal strength estimation subsystem and the display subsystem. The data storage and processing subsystem parses imported files of maps and base stations which are stored in the database and deals with them according to the need of the ray tracing algorithm. The signal strength estimation subsystem uses an estimation method based on forward tracing to calculate the signal strength distribution of the base station. The display subsystem shows imported data, processed data and 
estimation results by calling the MapInfo interface.

The second task of this paper is to improve the system operation results using processed the field strength distribution by centered moving average method and compare them with measurements [3].

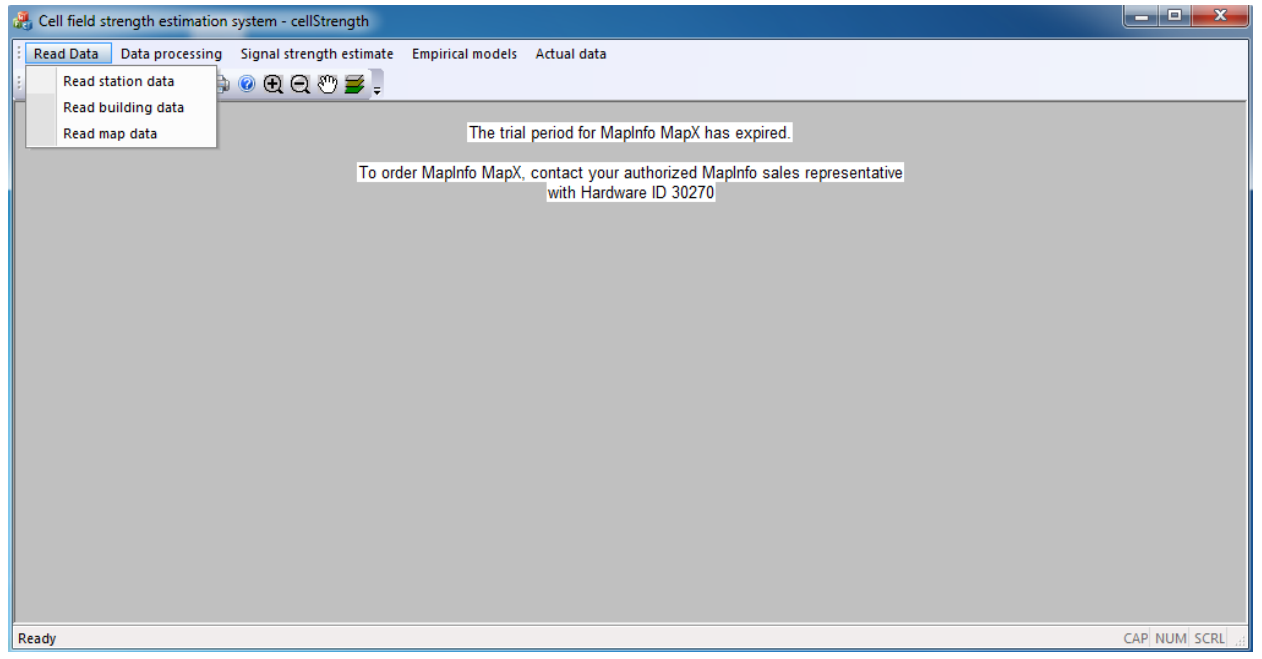

Figure1: Main interface of the system

\subsection{The geographical environment modeling}

The campus of Beijing University of Aeronautics and Astronautics is chosen as the study area, the range is $1305 \mathrm{~m}^{*} 1375 \mathrm{~m}$ using the mesh method, and the cell is divided into a $5 \mathrm{~m} * 5 \mathrm{~m}$ grids. This makes a total of $261 * 275=71775$ grids.

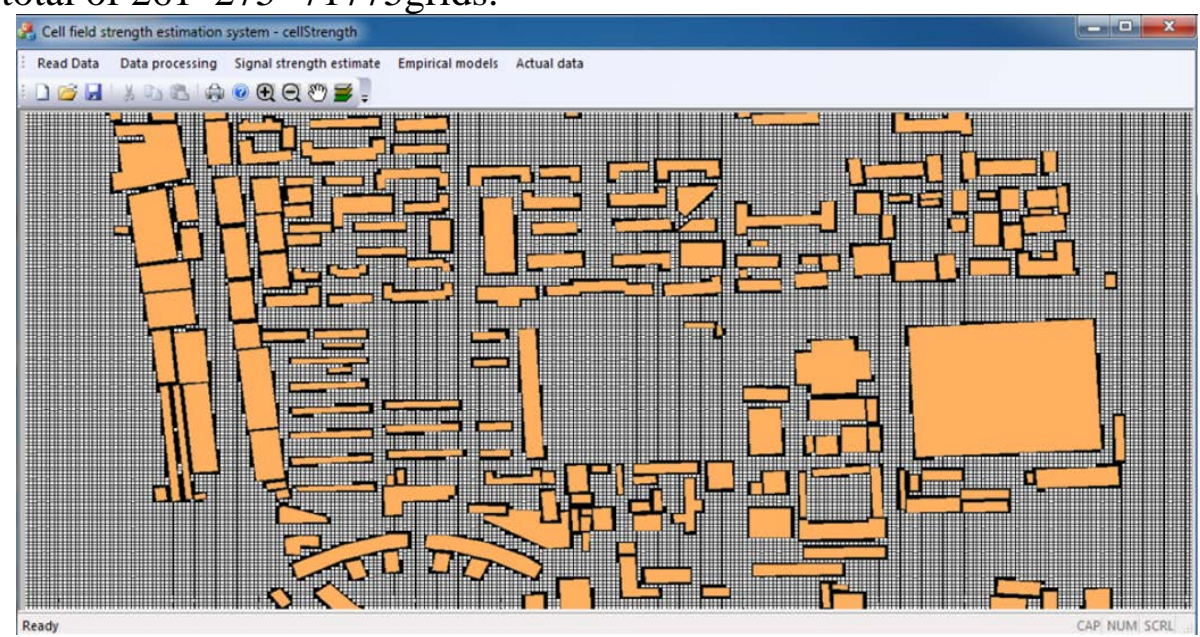

Figure 2: The study area is divided into a grid after a two-dimensional map

\subsection{System operating results}

The system operating results are ray tracing results which are the field strength values within the grid. The field is divided into several small power ranges represented by a different color. The base station is located in building $\mathrm{N}^{\circ} 3$ (blue dots) as shown in figure 3 , an antenna azimuth of 240 degrees, a decline angle of 8 degrees and the radiation frequency is $1,8 \mathrm{GHz}$. 


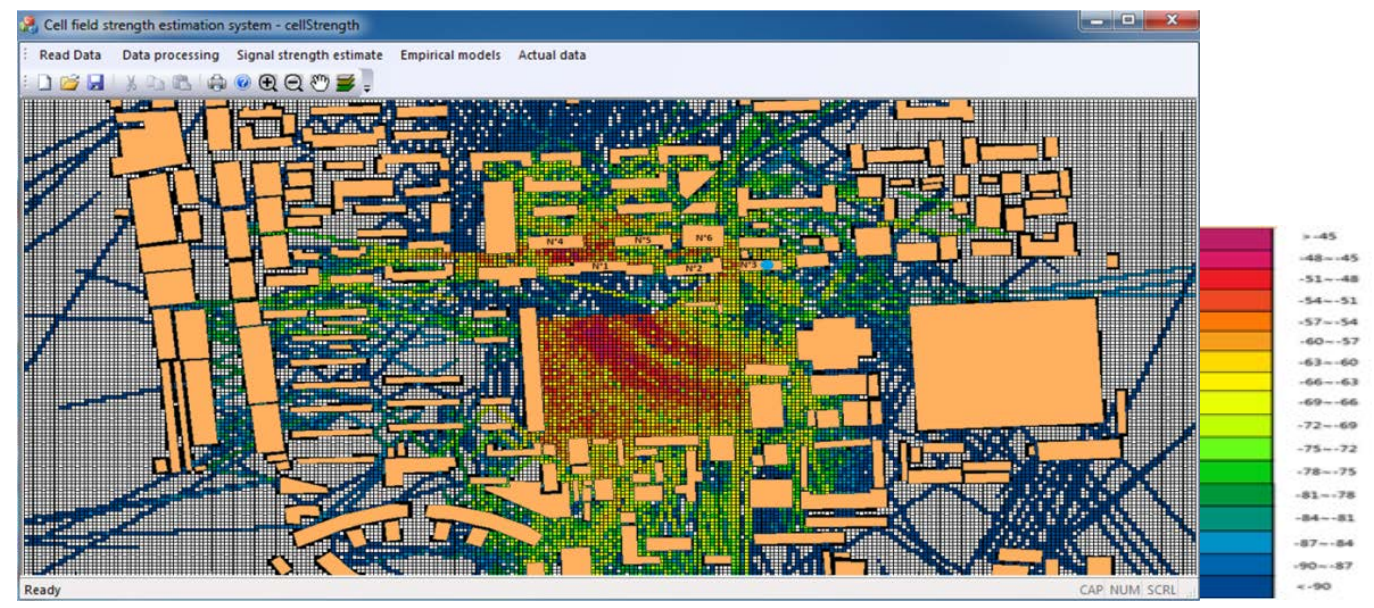

Figure3: Cell field strength estimate inside the campus based on ray tracing

\section{Field Strength Smoothing}

Recently, many authors have discussed various types of smoothing formulations in varying levels of generality (as far as the signal and observed process models are concerned) [4, 6]. Smoothing is an important aspect in the data processing to get important knowledge or processing outcomes with a good performance. In this paper, the processing we are discussing is field strength data smoothing using Moving Average algorithm [7, 8]. In using MA, we have to choose several points which will be averaged, for example data trend with 3 points average called as moving average with 3 orders or 3-MA smoother will be count in the observation. There are 4 types of Moving Average algorithms: Simple MA, Centered MA, Double MA, and Weighted MA. Here we are focusing on Centered Moving Average Algorithm (Centered MA).

\subsection{Centered Moving Average Algorithm (Centered MA).}

The centered moving average method consists of computing an average of an $n$ period data and associating it with the middle of the period interval. Here in our case we choose $n$ grid then we calculate the average of the signal strength values of these grids and place it to the mid-period $\mathrm{Eq}(1)$. We assume $n$ is odd:

$\widehat{F S}_{i}=\frac{\sum_{i}^{\frac{n-1}{2}} F S_{i-1}+F S_{i}+\sum_{i}^{\frac{n-1}{2}} F S_{i+1}}{n}$

$\widehat{F S}_{i}$ : Average field strength of the grid $i$.

$F S_{i-1}$ : Field strength of the grid $(i-1)$.

$F S_{i+1}$ : Field strength of the grid $(i+1)$.

\subsection{Results and discussion}

The ray tracing field strength results are processed or smoothed by centered moving averages, and results are as follows:

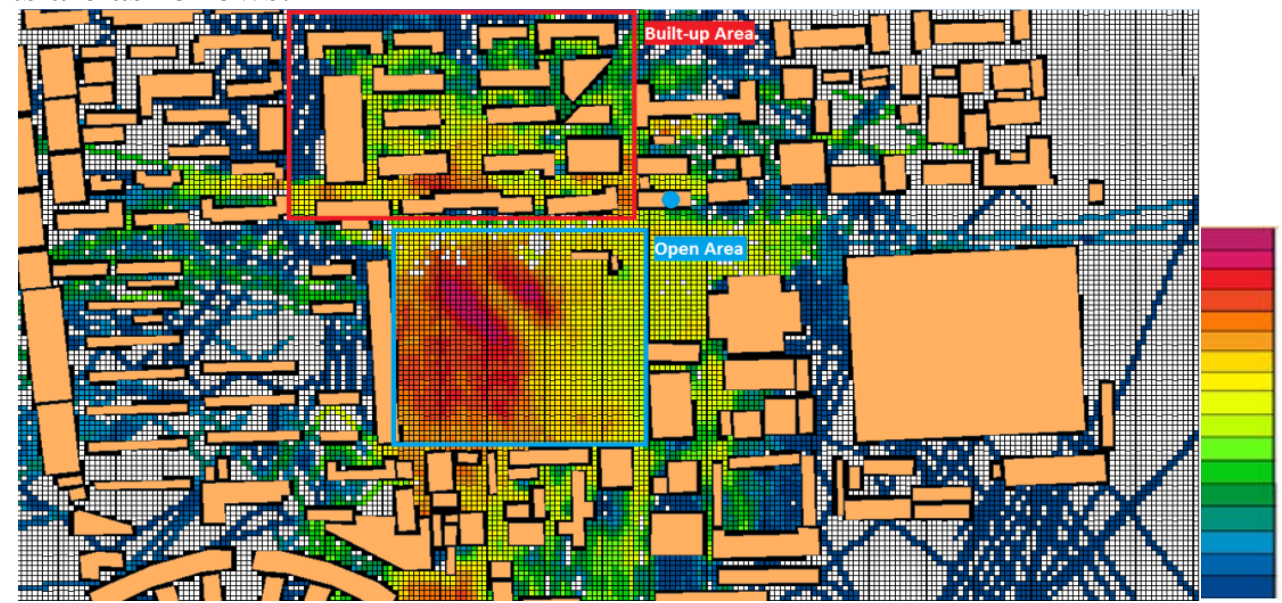

Figure 4: Smoothed field strength distribution 


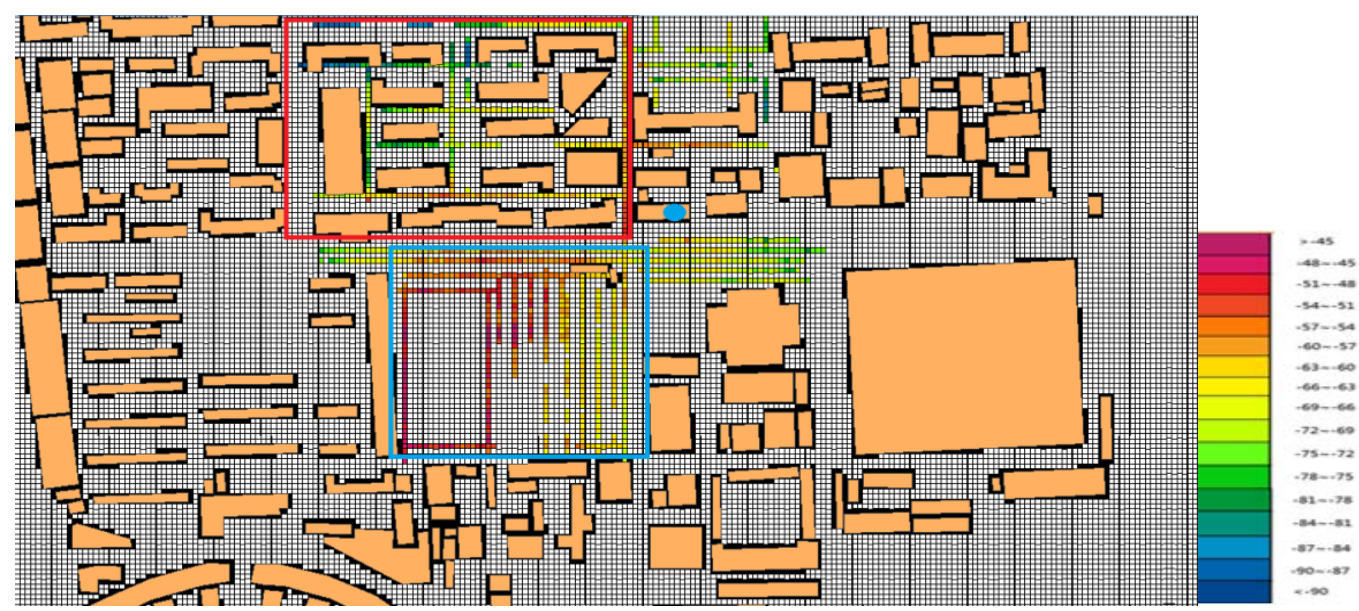

Figure 5 Measured field strength distribution

Comparing the smoothed field strength distribution with the measured data, we find that in both dense areas and open areas away from the buildings, they are good agreement.

\section{Conclusion}

Cell field strength estimating system software based on ray tracing is presented for outdoor environment. The theoretical basis of the model is three dimensional geometric optics, geometric optics GO and uniform theory of diffraction UTD. In addition, a smoothing algorithm is implemented for processing the field strength distribution by using centered moving average process, and the results show a good agreement with the measurements.

\section{Reference}

[1] Hata, M, “Empirical Formula for Propagation Loss in Land Mobile Radio Service,”. IEEE Transactions on Vehicular Technology, VT-29,3, p. pp. 317-32. 1980.

[2] K. Rizk, J.W, F. Gardiol, "Two-dimensional ray tracing modeling for propagation in microcellular environments”. IEEE Trans. Veh. Technol, vol. 46, p. 508-517. May 1997.

[3] Zhang Zhe, Zhang Jiebin, Zhou Xinxin, Gao Qiang”. Cell signal strength estimation method based on ray tracing [J]. Telecommunications 2016.5.

[4] T. Kailath and P. Frost. "An innovation approach to least-squares estimation. Part II: Linear smoothing in additive white noise.” IEEE Trans. Automat. Co ntr,. Vol. AC-13. pp. 655-660. 1968.

[5] D. G. Lainiotis. “An umfying framework for linear estimation: Generalized partitioned algorithms.” Inform. Sci., vol. 10. pp. 243-278. 1976.

[6] L. Ljung and T. Kailath. “A unified approach to smoothing formulas.” Automatica. vol. 12. pp. 147-157. 1976.

[7] Hyndman, R.J.: Moving Averages. International Encyclopedia of Statistical Science, pp 866869. Springer Berlin Heidelberg (2014).

[8] Raudys, A. Lenčiauskas, V. \& Malčius, Smoothing. Information and Software E.: Moving Averages forFinancial Data Technologies (403), 34-45. Springer Berlin Heidelberg (2013). 